\title{
Anesthetic challenges placed by Deep Brain Stimulation surgery in a child with severe Hallervorden-Spatz disease
}

M. Nunes Ferreira 1 , B. Matias ${ }^{2}$, P. Amorim ${ }^{3}$

${ }^{1}$ Anaesthesiology Department, Instituto Português de Oncologia Francisco Gentil do Porto - Porto (Portugal), ${ }^{2}$ Anaesthesiology Department, Centro Hospitalar de Setúbal - Setúbal (Portugal), ${ }^{3}$ Anaesthesiology Department, Centro Hospitalar do Porto - Porto (Portugal)

\section{BACKGROUND}

Hallervorden-Spatz disease is a rare neurodegenerative disorder with extrapyramidal dysfunction. Deep brain stimulation (DBS) of the basal ganglia is a high-risk treatment option. General anesthesia is mandatory, however it must be compatible with good quality electrical recording and free movements, a challenge in the case of a child.

\section{CASE REPORT}

A 9-year-old boy of $17-\mathrm{kg}$ with permanent generalized dystonic movements came for bilateral DBS. He required BIPAP at night, feeding through a PEG and while mentally sound he had lost manual control of an iPad and a joystick.

\section{INTRAOPERATIVE MANAGEMENT}

After standard ASA monitoring a Bilateral Bispectral Index (BIS) sensor was placed for raw and spectral EEG analysis. BIS was 98.

- Remifentanil was started at $0,13 \mu \mathrm{g} / \mathrm{kg} / \mathrm{min}$. BIS decreased to 60 and eyes closed.

- Propofol $1 \%$ was then started at $50 \mathrm{ml} / \mathrm{h}$ and infused until loss of the corneal reflex which occurred after $21 \mathrm{mg}$.

Propofol was stopped, ventilation started and intubation performed after $20 \mathrm{mg}$ of Rocuronium. BIS remained between $40-60$ with the spectrogram showing the normal Propofol pattern.

Propofol was then resumed and titrated to BIS.

When deep-brain electrical recording started, BIS was maintained very close to 60 .

For the stimulation phase:

- 50mg Sugammadex reversed NMB;

- Remifentanil infusion was doubled.

During both recording and stimulation there

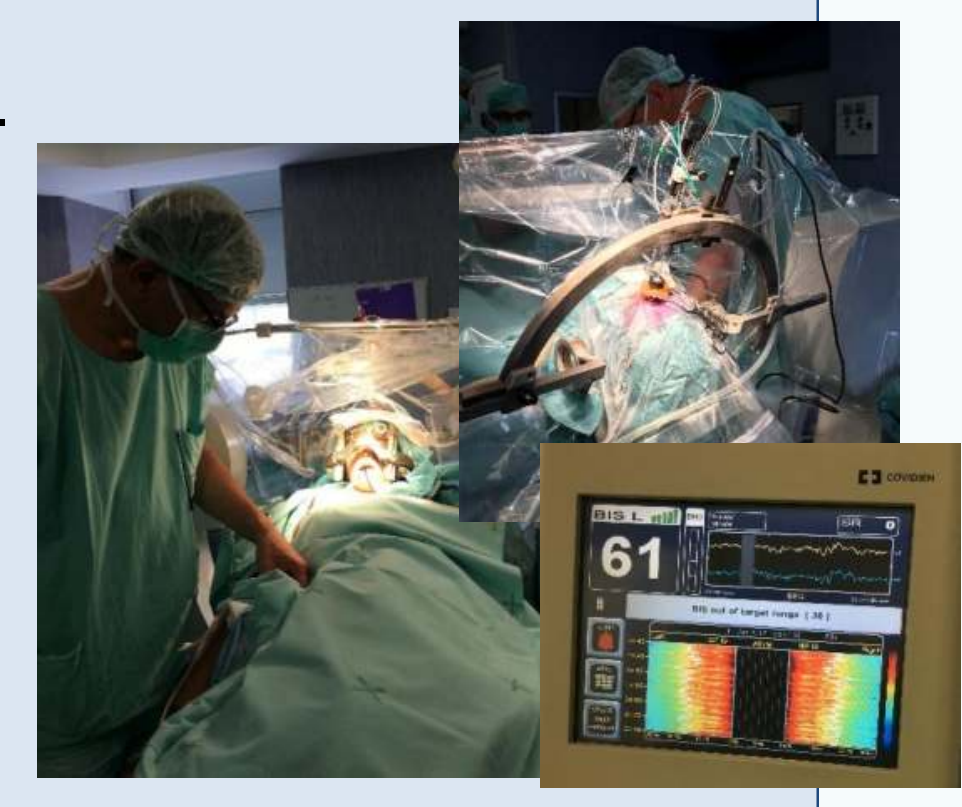
was no reaction to the tube, recordings were of excellent quality and movement could be observed when stimulus intensity was increased. This allowed the surgical plan to be performed without complications.

Total duration of procedure: 587 minutes.

Average Propofol was 3,6mg/kg/h and Remifentanil $0,12 \mu \mathrm{g} / \mathrm{kg} / \mathrm{min}$.

Ten minutes after the end of surgery the patient woke up and was extubated without complications.

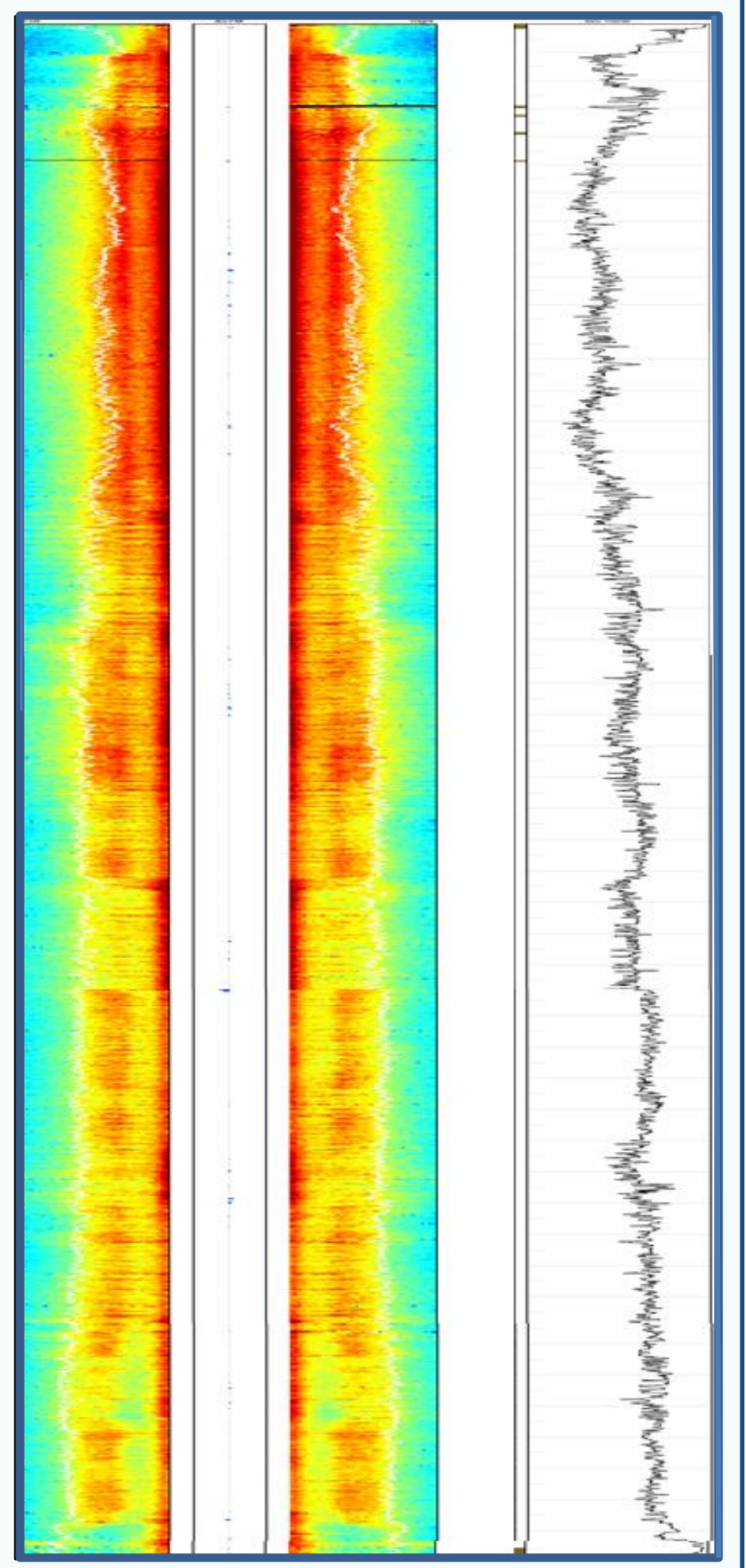

After the procedure, the patient was transferred to Pediatric ICU. On day 1 the battery was turned on and on day 3 the child was already capable of eating by mouth and using his iPad.

\section{DISCUSSION}

This case shows that intraoperative deep brain electrical recording and observation of movement during DBS surgery may be compatible with general anesthesia in a child.

Using EEG monitoring and the corneal reflex were key to titrate anesthesia. Remifentanil permitted tube tolerance without muscular paralysis. Our management differed significantly from the two published reports ${ }^{1,2}$. In this context, it may be useful to help in the management of future cases.

EEG and NMB monitoring and rapid acting Propofol, Remifentanil and Sugammadex, may allow general anesthesia compatible with DBS surgery in a child. 